\title{
Highlights from ABC5: Bridging the Gap
}

\author{
Rachel Wuerstlein $^{a} \quad$ Carlos Barrios $^{b} \quad$ Matteo Lambertini ${ }^{c, d} \quad$ Janice Tsang ${ }^{e}$ \\ Joana M. Ribeiro ${ }^{f}$ Renate Haidingerg \\ ${ }^{a}$ Breast Center, Department of Obstetrics and Gynecology, University of Munich (LMU) and CCC Munich LMU, \\ Munich, Germany; ${ }^{\text {b Centro de Pesquisa em Oncologia, Hospital São Lucas, Porto Alegre, Brazil; ' } D e p a r t m e n t ~ o f ~}$ \\ Medical Oncology, U.O.C. Clinica di Oncologia Medica, IRCCS Ospedale Policlinico San Martino, Genova, Italy; \\ ${ }^{\mathrm{d}}$ Department of Internal Medicine and Medical Specialties (DiMI), School of Medicine, University of Genoa, Genoa, \\ Italy; ${ }^{\mathrm{e}} \mathrm{Li}$ Ka Shing Faculty of Medicine, The University of Hong Kong, Hong Kong Breast Oncology Group, Hong

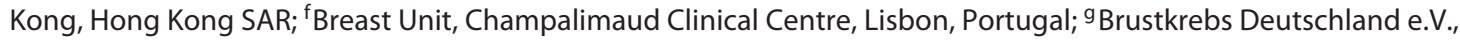 \\ Hohenbrunn, Germany
}

The International Consensus Conference for Advanced Breast Cancer $(\mathrm{ABC})$ has established itself as the major international conference for advanced breast cancer $(\mathrm{ABC})$. Its primary goal is the development of international consensus guidelines for the management of $\mathrm{ABC}$ patients. These guidelines are based on the most up-to-date evidence and can be used to guide treatment decision making in many different health care settings globally, with the necessary adaptations due to different access to care.

More than 1,500 experts from all over the world health professionals and patient advocates - attended ABC5 in Lisbon (Portugal) on November 14-16, 2019, chaired again by Fatima Cardoso, Lisbon. This conference was followed by the second ABC Global Alliance Meeting on November 16-17. The ABC conferences also aim to identify research priorities based on the most important areas of unmet needs, analyze and discuss available data to provide the most accurate management recommendations, as well as influence policy makers and funding bodies and ultimately improve standards of care, survival, and quality of life (QoL). Research and education, with accurate usage of available knowledge, throughout the world are key to achieving these goals.

Further information concerning ABC5 is available from: www.abc-lisbon.org.

In this Expert Discussion, 5 experts (including 2 panelists) with different backgrounds and perspectives) give insights into their take-home message from $\mathrm{ABC} 5$ and its consensus transfer to the different continents.

\section{Question 1: From ABC4 to 5 in 2 Years, What Have Been the Most Clinically Relevant Changes in Your Daily Practice in Metastatic Breast Cancer (MBC)? Please Focus on MBC Generally, and Then Subtypes Luminal, HER2+, and Triple-Negative Breast Cancer}

Barrios: The most clinically relevant changes since $\mathrm{ABC} 4$ refer to the profound understanding of the heterogeneous nature of cancer when we apply biology to a specific patient. The need for understanding the molecular determinants of a specific patient or tumor represents both a challenge and an avenue for directing further research. Although not all advances are aligned with the concept of heterogeneity and patient subgroup selection, as we evolve, most of the improvements will be related to benefits directed to biomarker-selected subgroups.

Overall, one revolutionary concept we will need to address is that hormone receptors (HRs) and HER2, as currently defined by IHC, represent targets that help in directing therapy rather than particular disease subgroups. Available molecular profiling clearly indicates that subgroups of diseases can be identified within each of these categories. However, the clinical usefulness of molecular classifications remains an unmet need and a research priority.

As an exception to this rule, the introduction of CDK4/6 inhibitors is certainly revolutionary for most if not all patients with $\mathrm{HR}+$ disease and changes the approach to this disease in clinical practice. After almost 40 years of single-agent endocrine therapy (ET), we came to 
realize that the interaction of endocrine signaling with other cellular pathways is important and has transforming clinical impact. Further developments in this area have already been presented and will certainly lead to further identification of other subgroups (i.e., PIK3CA-mutated patients responding to alpelisib in second-line therapy) within the HR+ population that will require more personalized approaches.

We have clearly demonstrated that advanced HER2+ disease patients certainly benefit from double blockade strategies as well as from the sequencing to T-DM1 followed by other combinations containing anti-HER2 drugs, resulting in significant benefits in overall survival (OS) with very long-term follow-up results available.

In advanced triple-negative breast cancer, the introduction of immunotherapy in patients with PD-L1-positive tumors and the benefits of PARP inhibitors in $B R$ $C A$-mutated patients are very encouraging advances. However, as with any improvement new questions arise, and as an example, selection of the best first-line treatment for patients with both biomarkers remains an issue. Furthermore, as has been seen in other tumor types with immunotherapy, the progression-free survival (PFS) benefit is proportionally modest compared to the magnitude of the OS differences, and it will be important to follow these patients for long-term survival as the hope for more profound benefits may emerge for certain patients.

Lambertini: After $\mathrm{ABC} 4$, new targeted treatments, i.e., CDK 4/6 inhibitors, PARP inhibitors, and immune checkpoint inhibitors, have been shown to both improve $\mathrm{OS}$ and $\mathrm{QoL}$ in patients with $\mathrm{ABC}$. These new findings, in addition to the recent progress in survival outcomes obtained in the HER2+ setting with pertuzumab and T-DM1, have changed the way oncologists evaluate clinical endpoints in the advanced setting, making them less willing to accept treatment-related toxicities in the absence of a clear survival gain.

\section{Question 2: Regarding the Consensus of Saturday at ABC5: Was There Any Vote Not according to Your National Guidelines/Standards of Care or Any Result with Which You Disagree with the Panel's Vote?}

Haidinger: Proper PD-L1 testing for triple-negative breast cancer patients and germline genetic testing for eligible patients are now very important as they have an impact on therapeutic decisions.

Barrios: Coming from a developing country, the most important disagreement is not related to a specific vote or a recommendation per se, but to the challenge of availability and access. While this is important in developed countries as well, as we improve results the access discus- sion will become more and more important, reaching collective ethical and moral dilemmas that will be at the center of future debates. Mostly, it is not a question of disagreement with a specific recommendation but the ability to offer all patients the best therapeutic option. The uncomfortable truth we all need to address is that currently, the vast majority of patients throughout the world only have limited chances of having access to optimal treatment and clinical trials.

Lambertini: In the ABC5 consensus session, $87.8 \%$ of panelists voted in favor of performing $B R C A$ genetic testing as soon as possible in all patients with $A B C$ for whom PARP inhibitors would be a possible option. By contrast, the Italian Society of Medical Oncology (AIOM) guidelines currently recommend $B R C A$ genetic testing only in patients fulfilling specific criteria with regard to personal and family history as well as tumor biology. However, based on the available data with the use of PARP inhibitors, $B R C A$ genetic testing has now important therapeutic implication; therefore, as suggested by the ABC5 panelists, this test should now be offered to all patients for whom PARP inhibitors would be a possible option. Since PARP inhibitors are now available in Italy, $B R C A$ genetic testing in patients with $\mathrm{ABC}$ needs to be implemented.

Tsang: Academically, I personally agree with the understanding of the panels vote at the ABC5 consensus. However, when we are serving back in our home country and hometown, especially in Asian countries, we have a wide spectrum of cities and countries with diverse economic backgrounds - from very rich and metropolitan cities or countries to much underprivileged cities and countries. While many of the anticancer agents are still self-financed items in Hong Kong and other parts of Asia, so are the diagnostic tests, including the companion diagnostics.

\section{Question 3: Are There Changes in the Combination of Local and Systemic Treatment in MBC (i.e., Oligometastatic Disease)?}

Barrios: This is an increasingly important issue. We are progressively changing the ability to stage our patients. Among other technologies in development, the availability of PET scans, sometimes with specific biomarkers and the use of ctDNA analysis, will enable us to identify disease at different and much earlier stages.

The current definition of oligometastatic disease suffers from the fact that we could be detecting low-volume disease either because the pace of the disease is slower and we are performing a test earlier in time or because we are getting better at detecting it with more sensitive technologies. The increasing numbers of patients in this scenario and the improvements in both local strategies (i.e., local- 
ized radiotherapy techniques) and systemic therapies will certainly lead to further research in this area. In this regard, and giving support for exploring this line of research, it is important to consider that we have already proven that systemic therapy is effective in micrometastatic disease when applied in the adjuvant setting.

Ribeiro: During ABC5, we have seen data that reinforce the notion that oligometastatic breast cancer differs from multimetastatic disease in prognosis and survival. Experts underscored the need for an individualized, multidisciplinary management of oligometastatic breast cancer with the intent being to cure some patients. Decision on the treatment of oligometastatic breast cancer (either the primary or metastasis-specific local) is nonetheless controversial given the sparse data.

Until that time, the decision to undergo locoregional treatment (either of the primary or the metastasis-specific local) must be decided on an individual basis, acknowledging that a small, but important, subset of patients with $\mathrm{ABC}$ can achieve complete remission and a long-term survival.

\section{Question 4: Prognosis for Young Patients in EBC Has Improved. What Needs to Be Done to Achieve Better Outcomes in Young Patients ( $<40$ Years Old) with MBC?}

Haidinger: Premenopausal women should be more included in trials, as there seems to be no substantial difference. Side effects (short-term and long-term) become more relevant with extended OS.

Lambertini: In the last years, randomized trials specifically dedicated to young premenopausal patients with advanced disease have finally been performed. The main takehome message is that no major difference should be expected in treatment benefit and outcomes compared to those observed in older patients. Therefore, there is no reason to exclude these patients from trials testing new anticancer treatments as was often done in the past. Thanks to major improvements in the survival outcomes of patients with advanced disease also among young premenopausal women, a growing importance should now be paid to their QoL and the potential long-term negative consequences of the proposed anticancer treatments. Among them, fertility and pregnancy-related concerns as well as sexual health are of particular relevance for young women; according to the $\mathrm{ABC}$ guidelines, these are relevant issues to be discussed and managed also in patients with $\mathrm{ABC}$.

Ribeiro: Available data do not suggest a specific medical treatment approach for young patients with breast cancer. However, it is clear that breast cancer in young women is enriched with aggressive subtypes. Triple-negative breast cancer disproportionately affects young women. In this setting a more generalized access to BRCA1/2 germline testing and use of platinum and/or PARP inhibitors will likely improve the outcomes. Knowledge about the impact of young age in other domains of antitumor immune response, given the unique characteristics of the breast milieu in which these tumors develop and the key role of estrogens in regulating immune response, is lacking. These can have implications for immune strategies (combinatorial/others) and must be explored.

In recent years there have been more and more trials accommodating and inclusive of younger women. Nonetheless, guideline recommendations are generally based on data generated from studies in postmenopausal patients (see Breast Cancer in Young Women [BCY] consensus). It is clear that premenopausal patients should not be excluded from clinical trials. In the setting of luminal breast cancer and endocrine-based approaches, patients should be rendered postmenopausal by ovarian function suppression/ovarian ablation.

\section{Question 5: New Agents (PARP Inhibitors, Checkpoint Inhibitors, CDK 4/6 Inhibitors) Have Shown Impact on OS. How Will This Change Our Discussion of Clinical Meaningful Endpoints and Our Clinical Trial Designs?}

Haidinger: Again, PFS was discussed and the panel members could not understand that it is without acceptance except for German health care authorities. A long PFS is meaningful as long as there is a good QoL. Nonetheless, OS always should be aimed for.

Ribeiro: During the ABC5 conference, there was a lot of debate around optimal clinical endpoints/trial designs. Interesting data showed that preferences for endpoints (PFS/OS) may differ between stakeholders. Patients with $\mathrm{ABC}$ rated the importance of OS higher than health professionals.

Several ideas emerged from the discussions. OS is still the best endpoint, but to help patients access new drugs we need to power trials for relevant secondary endpoints. Given the multidimensional effects of interventions, clinical trials must incorporate several endpoints (QoL). Clinical trial designs/endpoints need to take in account tumor biology and therapy type/line. In an era of personalized medicine, we must focus more on target-driven approaches.

Atezolizumab use in triple-negative breast cancer was extensively discussed. IMpassion 130 co-primary efficacy endpoints included PFS and OS in intention-totreat (ITT) and PD-L1 population. The trial protocol specified that formal testing of statistical significance in the PD-L1-positive population could only occur if statistical significance was shown in the ITT population. 
For the ITT population, the combination modestly improved PFS but not OS.

A place of consensus across all stakeholders at $\mathrm{ABC} 5$ was the indication for the generalized use of QoL endpoints in trial designs. The ESMO magnitude of benefit scale does consider the patient's value and doctors must embrace it. Clinical trial design challenges posed by the characteristic genomic landscape of breast cancer will be addressed in the next question.

Tsang: It was at the ABC5 that reflections and data have shown that different stakeholders hold different views on the respective clinical meaningful endpoints. It is always understandable that patients with $\mathrm{ABC}$ rated the importance of OS higher than health professionals.

\section{Question 6: Precision Medicine/Next-Generation Sequencing (NGS) Became Part of Our Diagnostics in MBC. In Your Environment, Is NGS Routine in the Field of MBC? Where Do You See Advantages and Where the Need for Discussions?}

Barrios: Other than as a requirement for participation in selected clinical trials, NGS is far from routine in the management of $\mathrm{ABC}$ in most developing countries (or most of the world). Currently, the limited clinical benefits, the costs of testing, and the lack of access to targeted agents represent significant barriers for wider application of molecular sequencing techniques. Nevertheless, the future indicates that molecular diagnosis will be a fundamental and unavoidable aspect of the management of breast cancer patients. Selection of patients according to PI3K pathway alterations and the presence of $B R C A$ mutations are already part of our current practice guidelines. However, implementation of these recommendations represents a significant challenge and needs much improvement in most countries.

Lambertini: Despite the fact that precision medicine with the use of NGS is still mostly limited to clinical trials in my country, currently, a few genetic alterations have shown clear therapeutic implications (e.g. germline $B R C A$ mutations and somatic PIK3CA mutations) in ABC. Therefore, with more genomic alterations being therapeutically relevant, growing attention should be paid to this field, including the logistic and quality control behind the potential widespread use of these technologies.

Ribeiro: NGS is not routine in my institution. It is, however, increasingly important to ask if at least some patients with $A B C$ should undergo NGS. It is clear that it is only worth doing a test if it will change patient care. Routine care in clinical practice is performed with approved agents or by providing patients clinical trial options.
Outside well-known biomarkers we can anticipate scenarios with clinical promise targeting oncogenes like AKT1/ERBB2/ESR1 mutations or TRK fusions. However, no evidence exists, at this moment, that giving targeted therapy is better than standard of care. MOSCATO 01 and SHIVA clinical trials have shown that off-label use of molecularly targeted agents should be discouraged. ABC5 recommendation stated that NGS should not be used in patients with $\mathrm{ABC}$. It is important to explain to patients the limitations of NGS results.

Tsang: In the era of Precision Medicine, NGS is not routine in my institution or back in our routine clinical practice. Yet academically, it has become increasingly important and relevant to explore any potential new drug avenue. In terms of cost effectiveness, it is well understood that unless the test could help us identify a particular drug target with the drug available and comes with absolute clinical benefit, we do not recommend routine use of NGS. At the moment, precision medicine with the use of companion diagnostics is limited to the clinical trial or research setting, and not universal to all patients.

\section{Question 7: PRO Became a Major Issue in All Clinical Trials and the Real-World Data Sets and Clinical Settings. How Is Communication between Patients and Health Care Professionals Going to Change in the Next 5 Years? And How Can This Be Facilitated?}

Haidinger: PRO is a mandatory measurement nowadays and has become more and more important as it might differ from the perception of physicians. We need better validated questionnaires for $A B C$ as the existing ones do not differentiate between early and metastatic disease.

Barrios: As the control of $\mathrm{ABC}$ and the objective survival benefits have been improving, important issues such as PRO and the long-term consequences of treatment are becoming the focus of increasing interest. However, the collection of this information is currently mainly concentrated in clinical trials. The scales we use in research are not routinely applied in clinical practice where only a very subjective and inconsistent evaluation of specific toxicities is used in the management of patients.

In the near future, a more continuous follow-up facilitated by an almost constant ability to follow and instantaneously report on patients' symptoms and complaints using current web-based communication technologies will revolutionize our capacity to document and use this information, informing management decisions. Even though still considered second-class science, the collection of large amounts of information in the real-world scenario will emerge as an important complementary 
source of information that will have an important impact in shaping clinical practice recommendations.

Lambertini: PROs have proven to be crucial in accurately reporting patients' experience of treatment toxicities and QoL. These are particularly relevant outcomes in the advanced setting due to the need for long-term treatments. Importantly, the agreement between CTCAE criteria scores reported by clinicians and PROs in the context of clinical trials has shown to be only moderate, underlying the need for integrating PROs in the evaluation of adverse events, not only for research purposes but also in clinical practice. Efforts are ongoing, including those of the National Cancer Institute, to develop PRO-CTCAE measurement for use in clinical trials in conjunction with the standard CTCAE. The implementation of PROs in daily practice is warranted; technology (i.e., smartphones) is expected to be of great value in this regard.

\section{Question 8: "A Great Doctor Treats the Patient with the Disease" Was the Title of the Keynote Lecture. How Can We Train Our Teams to Treat Not Only the Disease, but Also the Patient?}

Haidinger: The wonderful Claire Myerson (patient with $A B C$ ) pointed out in her talk how important good communication skills are. Best and worst scenarios are asked for by the $\mathrm{ABC}$ patient community instead of median PFS or survival data. "The tumor has progressed" instead of "you have progressed" are examples on how to better communicate with patients. We need early inclusion of palliative care, explaining to the patient that they are the experts in treating cancer and/or treatment side effects, such as pain.

Ribeiro: $\mathrm{ABC}$ patients need great teams. Taking care of patients with $\mathrm{ABC}$ means facing a variety of different challenges and must be a team effort. The patient's journey is often long, faced with success and drawbacks, and filled with different players. Their families and/or friends also present their own needs, expectations, frailties, and doubts. Building team-based oncology care, bringing together clinicians, researchers, and patient advocates to discuss and define practical strategies for the organization of effective health care teams is crucial. Team-based care, relying on teamwork, task interdependence, backup behavior, team orientation, and shared problem solving and decision making will enable us to deliver a more efficient high-quality care. The importance of disciplinary diversity in team composition must be recognized. This concept centers on differences in function, education, and culture across the team members contributing to care. Understanding the skills and knowledge related to each team member is critical to optimize team's potential.
Teams must develop communication skills. Good communication skills are essential. Communication workshops are imperative and must call out all the intervenience. Meetings that bring together patients with $\mathrm{ABC}$, support groups, and health professionals designed to identify communication needs and gaps, but also where patients share care perspectives, will help health professionals to recognize their unique and different needs and expectations.

Tsang: We need to engage and train our future doctors to be holistic and humanistic doctors with passion and compassion. A listening heart is of paramount importance as after all, the general well-being of anyone is defined by the World Health Organization (WHO) to include physical, psychological, psychosocial, mental, and spiritual well-being.

This is even more relevant as, according to the Chinese culture, the basic unit is a family and the patient is always surrounded by many friends and colleagues and relatives. By being a humanistic doctor, listening to the unmet needs of the patient and trying our best to remind ourselves to be nonjudgemental could help enhance the wellbeing of the patient.

\section{Question 9: What Is the Most Clinically Relevant Information from ABC5 You Will Take into Account to Change Your Clinical Practice after the $A B C$ Conference?}

Barrios: Due to their impact on current practice and the fact that they pave the way for future research, a number of very important developments need to be acknowledged. Recognition that ET for HR+ breast cancer has changed forever is probably one of the most noticeable. Modulation of ET with CDK4/6 inhibitors, alpelisib, or mTOR inhibitors has certainly been established as the preferred strategy in different clinical settings. The heterogeneity of the biology of this subgroup of patients allows for the prediction that other combinations and other subgroups will be identified with further research.

Another critical development relates to the need for further and better diagnostic characterization of each tumor. This is another fundamental transforming concept that underlies most current and future research efforts and is likely to improve our current results even further.

Lambertini: Many relevant data have been discussed at ABC5. Among them, the need to screen all patients with triple-negative $\mathrm{ABC}$ for germline $B R C A$ mutations represents the most practice-changing information that will modify the way oncologists approach these patients. With PARP inhibitors now available for BRCA-mutated patients potentially leading to improved outcomes, particularly when used in the first-line setting, genetic testing should be performed as soon as possible after the diagno- 
sis of $\mathrm{ABC}$. However, regulatory and ethical concerns as well as costs represent important issues to face and overcome in the coming future.

Ribeiro: The main changes in $\mathrm{ABC}$ statements with clinical practice impact are related to CDK4/6 inhibitors and the immune checkpoint inhibitor atezolizumab. With the consistent OS benefit seen across CDK4/6 inhibitor trials (namely MONARCH-2 and MONALEESA 3 and 7) the consensus considered these agents as the standard of care for $\mathrm{HR}+\mathrm{ABC}$ in combination with $\mathrm{ET}$ in first- or second-line of therapy. Knowledge about PIK3CA and germline $B R C A$ mutational status was also underscored during $\mathrm{ABC} 5$ as it may drive subsequent therapeutic decisions. The previously discussed IMPASSION 130 results also changed the atezolizumab recommendation. Atezolizumab is now an option for first-line treatment in patients with PD-L1-positive triple-negative ABC.

Tsang: The most clinically relevant information from $\mathrm{ABC} 5$ includes the consolidation of the use of upfront CDK4/6 inhibitors in combination with ET in luminal $\mathrm{ABC}$, and this is not limited to postmenopausal patients but also menopausal patients or those with amenorrhea. The added value of CDk4/6 inhibitors is their use in all luminal $\mathrm{ABC}$ and the superiority over upfront chemotherapy in the absence of visceral crisis. However, there are still question marks on the optimal sequencing of ET with or without CDK4/6 inhibitors for a better response rate.

With regard to triple-negative breast cancer, the main changes in $\mathrm{ABC}$ statements with clinical practice impact are related to CDK4/6 inhibitors and the immune checkpoint inhibitor atezolizumab as these have become standard of care for luminal $\mathrm{ABC}$ and triple-negative $\mathrm{ABC}$ patients. Furthermore, knowledge about PIK3CA and germline $B R C A$ mutational status was also underscored during $A B C 5$ as it may drive subsequent therapeutic decisions with particular reference to luminal $\mathrm{ABC}$ or triple-negative disease. Furthermore, the previously discussed IMPASSION 130 results also changed the atezolizumab recommendation. Atezolizumab is now an option for first-line treatment in patients with $\mathrm{PD}-\mathrm{L} 1$-positive triple-negative $\mathrm{ABC}$.

\section{Question 10: What Do You Think Will Be the Most Important Difference in MBC in 2 Years' Time at ABC6?}

Haidinger: More and new markers and tests to predict response to treatments, new treatments, and hopefully once again prolonged life expectancy for $\mathrm{ABC}$ patients.

Barrios: In 2 years' time, further selection of patients, especially with a better understanding of resistance mechanisms, will continue to evolve. Increasingly, biology will inform our treatment approach, allowing for smarter treatment selection and better results. One of the aspects we all agree needs improvement is the more appropriate selection of sequencing strategies. What to give first and how to follow on progression is an important question that our current trials do not address in a definitive way. If we want to increase survival and control the disease for longer periods of time this is a critical question.

Lambertini: In 2 years' time, the molecular classification of $A B C$ will be enriched by new predictive biomarkers that will lead to the availability of more effective targeted treatments. Many new promising agents, including several antibody-drug conjugates, are currently in the late stage of clinical development not only for the HER2+ subtype but also for patients with triple-negative breast cancer. These agents are also likely to change the current definition of HER2 expression in breast cancer, starting to look at HER2-low tumors as a new different disease entity.

Ribeiro: Great expectations. The consensus panel in ABC6 will vote for tucatinib and trastuzumab deruxtecan in HER2+ and atezolizumab in triple-negative breast cancer. It will be very interesting to see how the concept of "maintenance therapy," after induction chemotherapy, evolves in luminal ABC. Most experts at ABC5 did not accept adding CDK4/6i to ET if patients had started with chemotherapy. Will we have a different vote in 2 years?

Tsang: (1) A continuous emerging list of new drug articles of all subtypes of ABC5; (2) matching science with affordability across clinical subtypes and increasing review and burden of financial toxicity; (3) the advent of new biomarker-driven strategies for $\mathrm{ABC}$ and the optimal sequence; and (4) de-escalation of therapy treatment for highly selected patients. Stating better communication and seamless communication with holistic care is of paramount importance.

Thank you to all 5 experts for this great insight into your take-home messages from ABC5. We will report again from $\mathrm{ABC} 6$ in 2 years' time.

\section{Participants}

Prof. Carlos H. Barrios, MD

Grupo Oncoclínicas

Centro de Pesquisa em Oncologia, Hospital São Lucas, PUCRS

Latin American Cooperative Oncology Group (LACOG) Coroados 837

Porto Alegre RS 9190580, Brazil

barrios@tummi.org

Prof. Matteo Lambertini

Department of Medical Oncology

U.O.C. Clinica di Oncologia Medica

IRCCS Ospedale Policlinico San Martino and University of Genova

Largo Rosanna Benzi 10

16132 Genoa, Italy

matteo.lambertini@unige.it
80

Breast Care 2020;15:75-81

DOI: $10.1159 / 000505900$
Wuerstlein/Barrios/Lambertini/Tsang/ Ribeiro/Haidinger 
Dr. Janice Tsang

Li Ka Shing Faculty of Medicine

The University of Hong Kong

Hong Kong Breast Oncology Group (HKBOG)

Room 825, 8/F Tower A (Pacific Link Tower), Southmark

11 Yip Hing Street, Wong Chuk Hang, Hong Kong, Hong Kong SAR

janicetsangmedon@gmail.com
Dr. Joana Ribeiro

Champalimaud Clinical Centre

Avenida Brasília

1400-038 Lisbon, Portugal

joana.ribeiro@fundacaochampalimaud.pt

Ms. Renate Haidinger

Brustkrebs Deutschland e.V.

Lise-Meitner-Strasse 7

85662 Hohenbrunn, Germany

renate.haidinger@brustkrebsdeutschland.de 